\title{
The reliability problems of reading RFID tags in logistics warehouse systems
}

\author{
Pawel Zajac*, and Stanislaw Kwasniowski \\ Wroclaw University of Science and Technology, 27 Wybrzeze Wyspianskiego st. 50-370 Wroclaw, \\ Poland
}

\begin{abstract}
The use of RFID (radio frequency identification) tags in logistic warehousing systems has been analysed. The research was carried out at the RFID measuring station at Wroclaw University of Technology. The test results for $125 \mathrm{kHz}$ tags in dynamic write and read tags in one or several variants are presented. The applications presented in the article are universal and apply in widely understood logistic systems. In particular, they define the area of their use in logistic storage systems, in particular the identification of tagged and conveyed craft units, forklift trucks / stackers, or manually. Test results may improve the reliability of the automatic identification subsystem in logistic storage systems.
\end{abstract}

\section{Introduction}

Tags are used more and more frequently in logistics warehouse systems, automatic identification systems and other systems as well. The authors developed a warehouse rack overload sensor as a safety element of warehouse racking systems whereby an active tag was used (patent pending). Cooperating with logistics centers, the authors have noticed the need to increase efficiency of logistics warehouse systems. The paper [1-4] discussed those issues in relation to bar codes. Luggage RFID at airports has been known for a long time, albeit conditions of work of a passive tag write/read system are different than those in which the airport systems work. Unfortunately, increasing the efficiency of internal transport does not work in a way that would speed up a conveyor or a forklift truck, whereas an automatic identification system is capable of self-adaptation. Antennas, which are part of the identification system Figure 1 and are installed in the so-called gates, have to read reliably and as fast as possible all moved and stored shipping units along with piece items placed on top of them.

This problem is also encountered in the process of collection of piece items with the use of a mobile tag scanner in the case of a long and unsuccessful search of shipments in a warehouse (collection time gets longer which is a waste of time in accordance with the lean management rules) [5,6]. That is why fast and reliable identification of shipping units in loading subprocesses is very important. The research on the reliability of reading and writing tags, whose results were presented in this paper, was conducted at a laboratory station in the Laboratory of Automatic Identification in Logistics Systems at the Wroclaw

*Corresponding author: pawel.zajac@pwr.edu.pl 
University of Science and Technology. The said results were of a universal nature and delineated the area of application of tags. Moreover, they were also checked during implementation work.

\section{Logistics warehouse system vs. RFID}

Warehouse operation can be divided into the following processes: receipt, storage, collection, release. According to the requirements of the processes, an automatic identification system is selected so as to be reliable [7]. In this case, this applies to the selection of the RFID tags (this issue was discussed in detail in $[8,9]$ ), i.e. tag type/frequency, coded information vector and information read/write conditions in line with the WMS (Warehouse Management System) standards. Once such tags have been placed in a closed warehouse system (Figure 1), they have to work reliably.

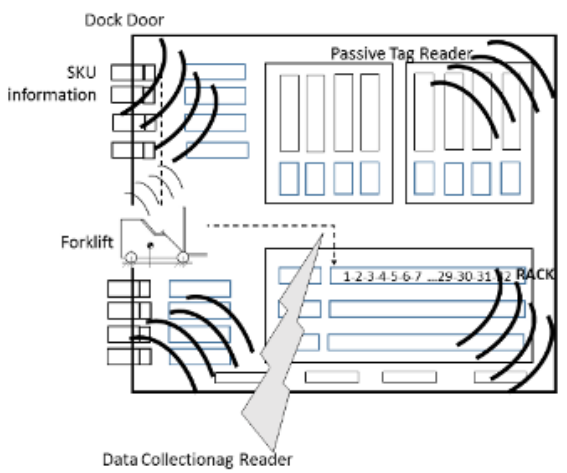

Fig. 1. RFID operation algorithm in the LSM (logistics warehouse system).

Activating a stationary antenna of an RFID reader or a mobile one in front of a full rack of found RFID pallets does not provide the answer to the question where the pallet sought for is - it is necessary to use functions and information collected in the WMS. However, it is not always possible, and it is certainly impossible in the case of a system failure. That is why, as part of safety improvements, non-collision read protocols and e-signatures are being introduced into the LSM. Issuing a read command in a place with a larger number of tags will cause many devices to send back a response to the base station at the same time. The individual pieces of information will overlap and the signal will eventually be unreadable. In order to avoid such situations, anti-collision algorithms are employed which are based on the mechanism of randomness. Waiting time of a base station is divided into so-called time slots. Transponders sending responses randomly choose a slot to transmit it in. This reduces the number of collisions that can occur, albeit it does not solve the problem completely. It may so happen that two transponders will draw the same slot. Philips has solved this problem using an 8-bit CRC (Cyclic Redundancy Check) algorithm. They are used in the WMS for navigating forklift trucks, e.g. determination of their whereabouts and operation status [10].

\section{Factors affecting reliability}

Experiments conducted under warehouse conditions allow spotting a problem with reliability of reading piece items. In the case of a cargo pallet with approx. 60-80 marked items, this reliability (or rather lack thereof) is not critical yet. However, when attempting to use RFID with a pallet with over a thousand items (e.g. books or pieces of clothing), the 
said reliability becomes critical. Currently existing tags and RFID readers are capable of reading approx. 300 tags per second. It would seem that it is enough then to stop under a gate with an antenna (a gate usually has several antennas: 2-5 pcs., this results from the radio wave coverage of the area of identification) for about 5 second in order for all tags to be read. Unfortunately, passive tags are powered by means of an query field of the antenna, there being no two-way communication between the two devices. Furthermore, a tag does not receive any information from the antenna that it can switch itself off already. In view of the above, tags closest to antennas will manage to response at least a dozen or so times, whereas tags further away sometimes do not response at all. The issue of determining electromagnetic characteristics of the packaging material becomes a major one as does the impact of placement and collection of items on pallets on its course. Actions aimed at increasing reliability can be expressed under three items: 1. Analysing the reliability of RFID systems, one comes across such terms as application Id (Id: identification). These are the terms which characterise the same mechanism consisting in communicating with a specific tag group only. A device activation command, sent from a base station, may contain a key which initialises communication only with those tags which have the same key saved in their memory. If a key received from a base station is different than that saved in the transponder, the device will remain silent. This type of tag identification is used in situations when items of various types are identified, i.e. items found in the same place (e.g. a warehouse) which are marked with identifiers containing different keys. Instead of reading all tags within the base station's coverage, only information from the tags that are of interest to us is received, i.e. from tags marked with appropriate keys; 2. Using authenticity checking mechanisms. Thanks to this, the data contained in a transponder are encrypted and can only be accessed by entering a correct password. Such mechanisms were employed, inter alia, in the TRIAS (triple radio automatic identification system) system. The principle of operation of this mechanism is based on a random number generator and an encryption key. A base station sends the number received from the random number generator to a transponder. Using the received number and its own identifier as the basis, the transponder generates a response. Subsequently, the transponder sends its own identifier and the generated response to the base station. On the basis of the previously generated number and the received identification number, the base station generates a series which should correspond to the response from the transponder. This completes the verification of authenticity of a tag; 3. Controlled access to information using tags with encryption of information. Two types of such devices are distinguished: tags with information protected during transmission and transponders with saved encrypted information. The principle of operation of those first ones consists in encrypting data before sending them from a transmitter and decrypting them once they have been received by the receiver. This technique is rarely used, since tags have to be equipped with encryption processors which raises the cost of devices and decreases the speed of their operation. The second group of transponders includes transponders with saved encrypted information. Once a base station has received encrypted information from a tag, it has to decrypt it using an appropriate key. This works similarly the other way round, i.e. in order for the base station to save information to the transponder, it first encrypts it with a key and the sends the data which are saved in the transponder's memory in encrypted form. Due to the necessity to perform encryption and decryption of data, the transmission gets slowed down.

\section{Dynamic tests}

The construction of the test station was shown in Figure 2a. A tag reader was connected with a computer. Tags were fixed to a rotating disc whose revolutions were controlled by means of an inverter. The inverter (T1) was connected with the rotating disc (T2) which 
transponders (T3) were mounted to. A straight edge was placed in close proximity to the rotating disc, in the tag's horizontal plane. The reader along with an antenna (reader) were placed over the rotating disc, but in such a way that only one tag was within the antenna's coverage. The placement of the reader relative to the tag was perpendicular. The position of the reader in relation to the rotating disc was being changed during measurements.

The set of alphanumerical characters used in writing tags corresponds to the set of MSI characters which was developed at MSI Data Corp. on the basis of the Plessey code. The MSI bar code, also called the modified Plessey code, is used mainly to mark racks with assortment. This code enables writing any number of digits in the $0-\mathrm{F}$ hexadecimal or the 0-9 decimal system, albeit the version for writing in the decimal system is used more frequently. The data vector has the following structure: start sign, any number of digits, checksum, stop sign. MSI uses either one or two digits to write the checksum. It can be calculated in several different ways and it depends on the application which way will be selected. For the purposes of this article, "modulo 10" has been chosen.

UniqueMaker v1.2 is a programme used to communicate with an antenna for reading/writing passive tags working at the frequency of $125 \mathrm{kHz}$, through the RS 232 interface. Next, the antenna gets connected to a computer in the programme which will automatically establish a connection with the antenna. A tag is then placed within the coverage of the reader's antenna. The programme displays the "read code" which is written in the tag. Finally, we enter a code to be written in the "New code" field and then click on "Programme", thus programming the tag. Experimental research plan: reading distance this test has been designed to check the maximum distance at which the transponder can be read by the reader; Mutual positioning (perpendicular or parallel) - this test has been designed to check the best positioning of the transponder relative to the reader's antenna; Tag movement speed relative to the reader - this test has been designed to check the maximum linear speed at which the tag can be read.

\subsection{Tag reading}

The test was to verify to what extend readings of several transponders collided. Constant angular speeds were determined when conducting the tests. The possessed devices were being checked several times for approx. $30 \mathrm{~s}$ after setpoints had been changed in order to stabilize the measurement conditions. In order to avoid measurement interference, the system was calibrated. The first tag was placed on the rotating disc with a diameter of 150 $\mathrm{mm}$. Linear speed was approx. $1 \mathrm{~m} / \mathrm{s}$. Reading distance was $40 \mathrm{~mm}$. Subsequently, the second tag and the third tag were added. At the outset of the measurements, test revolutions were performed in order to determine the range of revolutions. One tag was fixed to the disc which was then set in motion. After several attempts, the maximum number of revolutions was successfully established for tags operating at the frequency of $125 \mathrm{kHz}$, this value having been $140 \mathrm{rpm}$. Next, three tags at a distance of $180 \mathrm{~mm}$ from one another were mounted on the disc.

The disc with tags was set in motion and then it was established that the rotational speed was equal to $140 \mathrm{rpm}$. The reader was put closer to the rotating disc so that the center of the reader's antenna and the tag's center were aligned. Reading angle of $90^{\circ}$ was maintained. Each time no result was obtained, rotational speed was reduced. When a reading signal was obtained, the disc was stopped and the reading distance measured. 
a)

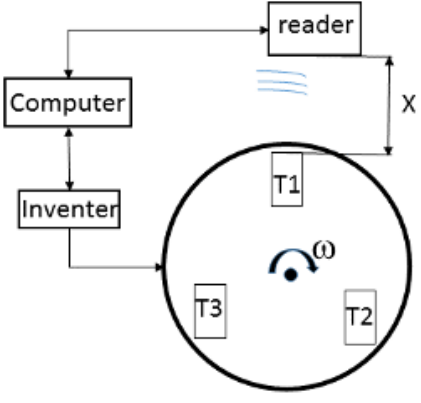

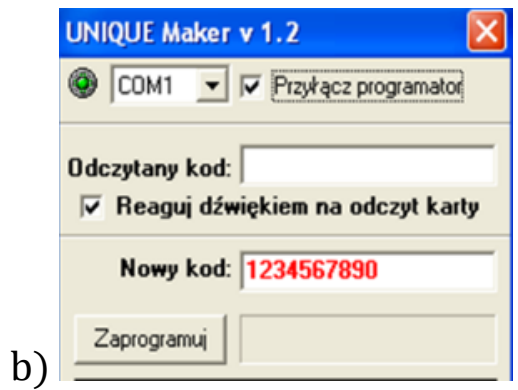

b)

Fig. 2. Diagram showing the measurement station of the UniqueMaker v1.2 programme.

Tests were also conducted for one and two tags, but mounted on a smaller arm. In order to calculate the linear speed, the following formula $V=\frac{2 \pi r n}{60}$ was used, where $\mathrm{V}-$ linear speed $[\mathrm{m} / \mathrm{s}], \mathrm{r}-$ disc radius $[\mathrm{m}], \mathrm{n}$ rotational speed $[\mathrm{rpm}]$. The obtained results were shown in Figure 3.

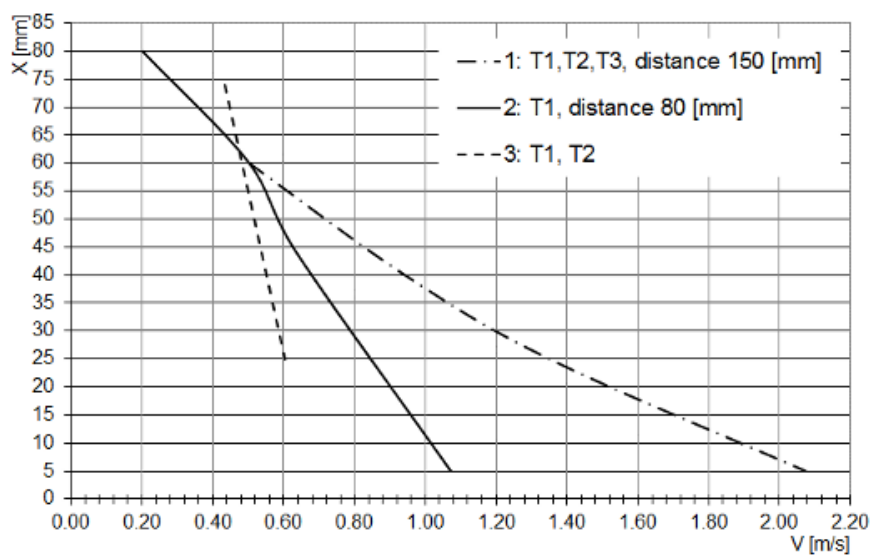

Fig. 3. Diagram for dynamic tests.

\subsection{Tag writing}

Stage of writing information in a tag, $1 \div 3$ pcs., simultaneously, during movement. In this case, 10 alphanumeric characters can be written (in line with the definition of the information vector above). Tag writing time is approx. $1.5 \div 2.0 \mathrm{~s}$. It is constant and does not change in the case of a change in distance of the tag from the programmer or the number of tags remaining within the antenna's coverage. Measurement 1. During programming, the transponder has to stay within the antenna's coverage. In order to ensure fully correct writing, the tag has to remain motionless. In the event of movement of the tag, writing may be incorrect. The programming time is approx. $1.5 \div 2.0 \mathrm{~s}$. In the case of failure to perform a correct write operation, the transponder will not be "visible" for the reader at all. Measurement 2. If there are two tags within the coverage of the programmer's antenna, one tag will be read (the one with better coverage). During writing, the same new code is written in both tags. Reading or writing will not possible if two tags are placed in their axis and are stuck together. In the case of two tags in the axis, the distance between them has to be at least $10 \mathrm{~mm}$. If the tags are stuck together, they have to be spaced in order to eliminate alignment. Measurement 3. Simultaneous writing of three tags within the antenna's 
coverage will take place if: three tags are placed axially, but perpendicularly, to the antenna and the distance between them is at least $15 \mathrm{~mm}$.

\section{Conclusions}

The optimum linear speed for the tags $(0.5 \mathrm{~m} / \mathrm{s})$ can be read from the graph in Figure 3. In this case, the number of tags or the mutual distance between them does not play any role. Furthermore, the smaller the speed, the higher the reading distance; and the higher the linear speed, the closer the reader has to be. This allows recommending these tags for use in logistics warehouse systems where it overlaps with the standard speed of roller conveyors. This is an important research proposal, universal in application.

The maximum reading distance is connected with the number of tags in their own vicinity. In the case of one tag, the reading distance $X$ is $80 \mathrm{~mm}$ at a speed of $0.2 \mathrm{~m} / \mathrm{s}$, whereas with two tags, the maximum reading is $74 \mathrm{~mm}$ at a speed of $0.4 \mathrm{~m} / \mathrm{s}$. With three tags, the reading distance decreases to $60 \mathrm{~mm}$ at a speed of $0.5 \mathrm{~m} / \mathrm{s}$. The maximum speed at which the tags are read is $2.1 \mathrm{~m} / \mathrm{s}$. However, in order to make use of such a high speed, the distance between the reader and the moving tag has to be very short. Series 2 includes one tag on an $80 \mathrm{~mm}$ arm, whereas series 3 includes two tags on the same arm; tags are $170 \mathrm{~mm}$ away from each other. It can be observed that the reading speed of two tags at the same speed decreases. This phenomenon occurs only over the speed of $0.63 \mathrm{~m} / \mathrm{s}$. It is the other way round at lower speeds, i.e. two tags are read faster, despite that intuition could suggest that it would be one tag that is read faster.

\section{References}

1. H. Bhatt, B. Glover, RFID Essentials (O'Reilly, 2006)

2. D. M. Dobkin, The RF in RFID. Passive UHF RFID in Practice (Elsevier, 2008)

3. K. Finkenzeller, RFID Handbook: Fundamentals and Applications in Contactless Smart Cards and Identification (John Wiley \& Sons, 2003)

4. A. Rizzi, R. Montanari, M. Bertolini, Logistica e tecnologia RFID (Springer, 2011)

5. A. Bujak, P. Zajac, Communication in Computer and Information Science, Telematics in the Transport Environment 329, 199-210 (Springer, 2012)

6. A. Bujak, P. Zajac, Communications in Computer and Information Science, Activities of Transport Telematics 395, 361-369 (Springer 2013)

7. A. Kierzkowski, T. Kisiel, J. Air Transp. Manag. (to be published)

8. S. Kwasniowski, T. Nowakowski, M. Zajac, Intermodal transport in logistics networks, (Publishing house of Wroclaw University of Technology, Wroclaw, 2008)

9. S. Kwasniowski, M. Zajac, P. Zajac, Communication in Computer and Information Science, Transport System Telematics 104, 391-399 (Springer, 2010)

10. P. Zając, Progress in Automation, Robotics and Measuring Techniques, 345-355 (Springer, 2015) 